Int. Journal of Math. Analysis, Vol. 8, 2014, no. 17, 813 - 827

HIKARI Ltd, www.m-hikari.com

http://dx.doi.org/10.12988/ijma.2014.4391

\title{
Estimators of Sensitivities of an Asian Option: Numerical Analysis
}

\author{
Abderrahmane Moussi \\ Department of Mathematics and Computer Science \\ Faculty of Sciences, University Mohammed I \\ 60000 Oujda, Morocco \\ Abdeluaab Lidouh \\ Department of Mathematics and Computer Science \\ Faculty of Sciences, University Mohammed I \\ 60000 Oujda, Morocco \\ Fatima Zahra Nqi \\ National School of Applied Sciences(E.N.S.A) \\ Faculty of Sciences, University Mohammed I \\ 60000 Oujda, Morocco
}

Copyright (c) 2014 Abderrahmane Moussi et al. This is an open access article distributed under the Creative Commons Attribution License, which permits unrestricted use, distribution, and reproduction in any medium, provided the original work is properly cited.

\begin{abstract}
In this paper, we investigate three methods for computing price sensitivities (or greeks) of an Asian option, namely, finite difference methods, a likelihood ratio method, and a pathwise method and we analyze the outputs. The efficiency of results is evaluated by means of Monte Carlo with and without, variance reduction technique.
\end{abstract}

Keywords: Asian Options, Monte Carlo, Variance Reduction, Finite Difference Method, Pathwise Method, Likelihood Ratio, Black and Scholes, Greeks, Price Sensitivities, Antithetic Variates 


\section{Introduction}

Asian options depend on the path of asset prices and their payoff is based on the average of the underlying asset prices over some period prior to maturity, which makes them interesting as hedging instrument,especially, in markets with strong movements. furthermore, they are very interesting to study whatsoever theoretically or practically. Indeed, These options are much more difficult to evaluate than the ordinary options and Standard techniques are often inconsistent, inaccurate, or require a lot of time. Practically, the payoff structure of Asian options makes them less vulnerable to manipulation. For the history and evolution of Asian options, one can refer to [8].

Several methods are used for pricing Asian options, we quote J.E.Zhang (2001) who proposed a semi-analytical method for pricing and hedging continuously sampled Asian options [10]. In [11], he presented analytical results up to the fourth order and demonstrated that the process converges quickly and gives accurate results. The method of "comonotonic approximations" has been developed by Kaas, Dhaene and Goovaerts (see Kaas et al. (2000)). An approach based on Taylor series approximations proposed by $N$.Ju [14]. For Monte Carlo simulation to price Asian options, we can name, for example, Kemna and Vorst (1990), or B.Lapeyre and E.Temam [4].

For hedging purposes, the estimation of the price sensitivities (Greeks) is often as important as the evaluation of the prices themselves. In this sense, Fourni et al. $(1999,2001)$ showed that under some conditions, rather general, a Greek can be represented as an expected value of the payoff multiplied by a certain weight function. Thus the Greek can be computed numerically by Monte Carlo simulation. The tools for computing the weight function are provided by the Malliavin calculus theory (see Benhamou [5], and Kohatsu-Higa and Montero (2004)). Note that the estimators obtained using the Malliavin method are not unique. One can obtain any number of different estimators for the same price sensitivity which may have significant differences in the variance.

In this paper, we discuss three methods of calculating Asian options Greeks without Malliavin calculus. indeed, standard mathematical method to calculate a derivative of securities, namely, the finite difference method using re-simulation has been treated by several authors, we quote Boyle et.al [16]. otherwise, two direct methods for estimating security price derivatives using Monte Carlo simulation are presented, a pathwise method and a likelihood ratio method in [13].

Given that closed form does not exist for the sensitivities of an Asian option, 
we will be brought to simulation which has proven to be valuable tool in this case. In particular, Monte Carlo Method, which is useful tool for many of these calculations. However, during last few years, efficiency and accuracy increased.

In this work, we analyze the three methods under different angles. Focusing on the accuracy and quality of the results. We propose, particulary, to improve the quality of results using variance reduction in the Monte Carlo framework. The body of this work will be organized as follows: The second section is devoted to three methods selected, that are finite difference methods, a pathwise method and likelihood ratio method applied to Asian options case. The third one to Monte Carlo method with variance reduction. In section 4, we give numerical results and comparisons. Finally, we conclude with some remarks.

Note that throughout this work, we restrict ourself to an Asian call option.

\section{Asian options sensitivities}

We consider Black and Scholes model, where risky asset is governed by the Stochastic differential equation (SDE):

$$
d S_{t}=S_{t}\left(r d t+\sigma d W_{t}\right), \quad t \geq 0,
$$

where $\left(W_{t}\right)_{(t \geq 0)}$ is a standard Brownian motion, and $r \geq 0, \sigma \geq 0$ are constants. The risky asset evolves under the risk neutral measure denoted by $\mathbf{P}$, and the unique solution of (1) is given by

$$
S_{t}=S_{0} \exp \left(\left(r-\frac{\sigma^{2}}{2}\right) t+\sigma W_{t}\right) . t \geq 0,
$$

The payoff of a discretely sampled arithmetic average Asian option is given

by $\max (\bar{S}-K, 0)$, where $\bar{S}=A_{m}=\sum_{i=1}^{m} S_{i} / m$, with, $S_{i}$ is the asset price at time $t_{i}$, and $T$ is the option maturity.

Under the risk neutral measure, the value of the option for given strike $K \geq 0$ is

$$
C=\mathbb{E}\left[e^{-r T} \max (\bar{S}-K, 0)\right] .
$$

Whereas prices for pricing securities can be observed in the market, their sensitivities to parameter changes typically cannot and must therefore be computed. Whence the importance of sensitivities in the hedging procedures. These sensitivities are also called Greeks because each of them is associated with a Greek letter. 
The most important for hedging purpose are the delta and Gamma. Indeed, on one hand, delta measures the rate of change of option value with respect to changes in the underlying asset's price, and provides information on the quantity of the underlying asset necessary to balance the hedge portfolio. On the other hand, Gamma of an option is the second derivatives with respect to the initial price of the underlying security, and it is related to the optimal time interval required for re-balancing a hedge.

Now, we define the Greeks which are the option price sensitivities relative to the underlying asset price. In fact, delta $(\Delta)$ is the first derivative of the value of the option with respect to the underlying instrument's price, while Gamma $(\Gamma)$ is the second derivative of the value of the option with respect to the underlying instrument's price. Vega $(\nu)$ and Rho $(\rho)$ measure, respectively, the sensitivity of an option price to a change in volatility, interest rate. Whilst Theta $(\Theta)$ reflects the rate of decline in the value of an option due to the passage of time.

In Black and Scholes model, if $C$ denotes the option price, and $S_{0}$ is the current underlying price, greeks are defined mathematically by

- Delta $(\Delta)$ :

$$
\Delta=\frac{\partial C}{\partial S_{0}}
$$

- $\operatorname{Gamma}(\Gamma)$ :

$$
\Gamma=\frac{\partial^{2} C}{\partial S_{0}^{2}}
$$

- Rho $(\rho)$ :

$$
\rho=\frac{\partial C}{\partial r}
$$

- Vega $(\nu)$ :

$$
\nu=\frac{\partial C}{\partial \sigma}
$$

where, $r$ is the risk-free rate, and $\sigma$ is the volatility.

Note that, in this paper we not deal with theta. Indeed, Theta is not the same type of hedge parameter as delta. There is uncertainty about the future stock price, but there is no uncertainty about the passage of time. It makes sense to hedge against changes in the price of the underlying asset, but it does not make any sense to hedge against the passage of time. In spite of this, many traders regard theta as a useful descriptive statistic for a portfolio. This is because in a delta-neutral portfolio theta is a proxy for gamma [12]. 


\subsection{Finite difference approximations}

This is the basic approach to estimate greeks. It is based on re-simulation to have price at the perturbed parameter. Here, we present delta case and let the reader infer the case of other Greek by analogical reasoning. To estimate delta, we simulate the price $C\left(S_{0}\right)$ and $C\left(S_{0}+\epsilon\right)$, thus the estimator of Delta is

$$
\tilde{\Delta}=\frac{C\left(S_{0}+\epsilon\right)-C\left(S_{0}\right)}{\epsilon} .
$$

We can compute $N$ independent copies of $\tilde{\Delta}$. By using $N \rightarrow \infty$ (by means of Monte Carlo method), the mean of these copies converges to the true finitedifference ratio, which is

$$
\Delta=\frac{C\left(S_{0}+\epsilon\right)-C\left(S_{0}\right)}{\epsilon}
$$

Glynn showed in [18] that the best possible convergence rate $\epsilon$ is typically $N^{-1 / 4}$. Replacing the forward finite difference by the central difference

$$
\frac{C\left(S_{0}+\epsilon\right)-C\left(S_{0}-\epsilon\right)}{2 \epsilon}
$$

improves the optimal convergence rate to $N^{-1 / 3}$. Also, Boyle et al. shows in [16] that in some cases the rate of convergence of this method is $N^{-1 / 2}$, especially through using common random numbers. Nevertheless, this method within the Monte Carlo framework suffer from two shortcomings: it is biased and they require multiple re-simulations [17]. Thus, we have two numerical errors, the first results from the use of finite difference approximations, while the second is related to the method of Monte Carlo. Note that for using this approach to compute Gamma, we have to re-simulate twice because the estimator can be written as

$$
\frac{C\left(S_{0}+\epsilon\right)+C\left(S_{0}-\epsilon\right)-2 C\left(S_{0}\right)}{\epsilon^{2}} .
$$

In what follows, we present the results for the two other chosen simulation methods for the calculation of greeks relative to an Asian option averaged arithmetically. These approaches are known are direct methods based on unbiased estimates for Asian option greeks.

\subsection{Pathwise Derivatives and Likelihood Ratios method}

In this paper we restrict ourself to expose the estimators values and invite the reader to consult [13] or [17] for more details. The pathwise method is based on the relationship between the security payoff and the parameter of 
interest. Differentiating this relationship leads, under appropriate conditions, to an unbiased estimator for the derivative of the security price. In contrast, the likelihood ratio method is based on the relationship between the probability density of the price of the underlying security and the parameter of interest. These estimates require Monte Carlo simulation. In the case of Asian options based on arithmetic average as defined above, the estimators of price sensitivities can be written as follows:

- Asian option pathwise derivative estimators :

$$
\begin{gathered}
\hat{\Delta}_{p}=e^{-r t} \mathbf{1}_{\{\bar{S} \geq K\}} \frac{\bar{S}}{S_{0}}, \\
\hat{\Gamma}_{p}=e^{-r t}\left(\frac{K}{S_{0}}\right)^{2} m g\left(S_{m-1}, w_{m}, \Delta t_{m}\right), \\
\hat{\nu}_{p}=e^{-r t} \mathbf{1}_{\{\bar{S} \geq K\}} \frac{1}{m \sigma} \sum_{i=1}^{m} S_{i} \times\left(\ln \left(\frac{S_{i}}{S_{0}}\right)-\left(r+\frac{1}{2} \sigma^{2}\right) t_{i}\right), \\
\hat{\rho}_{p}=e^{-r t} \mathbf{1}_{\{\bar{S} \geq K\}}\left(\frac{1}{m} \sum_{i=1}^{m} S_{i} t_{i}-T\right),
\end{gathered}
$$

where $\Delta t_{i}=t_{i}-t_{i-1}, w_{m}=m(K-\bar{S})+S_{m}, g(u, v, t)=n(d(u, v, t)) /(v \sigma \sqrt{t})$ with $n($.$) represents the standard normal density function,$ and $d(u, v, t)=\left(\ln (v / u)-\left(r-1 / 2 \sigma^{2}\right) t\right) /(\sigma \sqrt{t})$. Note that one can replace the estimator of gamma in (6) by an "hybrid" biased estimator, based on a resimulation of the pathwise delta estimator, which is

$$
\hat{\Gamma}_{h}=\frac{1}{\epsilon}\left(e^{-r t} \mathbf{1}_{\left\{\bar{S}\left(S_{0}+\epsilon\right) \geq K\right\}} \frac{\bar{S}\left(S_{0}+\epsilon\right)}{S_{0}+\epsilon}-e^{-r t} \mathbf{1}_{\left\{\bar{S}\left(S_{0}\right) \geq K\right\}} \frac{\bar{S}\left(S_{0}\right)}{S_{0}}\right) .
$$

Taking into consideration the optimal value to be taken for the value of $\epsilon$ [13]. Note also that One can use the estimators of gamma and rho defined in [17] by P.Boyle, and A.Potapchik, which are different from those given in [13]. In fact, we write :

$$
\begin{gathered}
\hat{\Gamma}_{b}=e^{-r t} \mathbf{1}_{\left\{A_{m} \geq K\right\}} \mathbf{1}_{\left\{C_{m}(K) \geq 0\right\}}\left(\frac{A_{m}}{S_{0}}\right)^{2} \times \frac{1}{\sigma \sqrt{h}} \frac{m}{C_{m}(K)} \phi\left(R_{m}(K)\right), \\
\hat{\rho}_{b}=e^{-r t} \mathbf{1}_{\left\{A_{m} \geq K\right\}}\left(A_{m}^{1}-T\left(A_{m}-K\right)\right),
\end{gathered}
$$

where $A_{m}$ is the arithmetic average of underlying asset price, $\phi$ is the cumulative normal distribution function. 


$$
\begin{aligned}
& h=t_{i}-t_{i-1}, \quad \text { and } C_{m}(K)=m K-\sum_{i=1}^{N-1} S_{i}, \\
& \text { finally, } R_{m}(K)=\frac{1}{\sigma \sqrt{h}}\left(\ln \left[\frac{C_{m}(K)}{S_{m-1}}\right]-r h\right), \text { and } A_{m}^{1}=\frac{1}{m} \sum_{i=1}^{m} t_{i} A_{i}
\end{aligned}
$$

- Asian option Likelihood Ratio derivative estimators :

$$
\begin{gathered}
\hat{\Delta}_{l}=e^{-r t} \max (\bar{S}-K, 0) \frac{1}{S_{0} \sigma^{2} \Delta t_{1}} \times\left(\ln \left(\frac{S_{1}}{S_{0}}\right)-\left(r-\frac{1}{2} \sigma^{2}\right) \Delta t_{1}\right), \\
\hat{\Gamma}_{l}=e^{-r t} \max (\bar{S}-K, 0) \frac{d_{1}^{2}-d_{1} \sigma \sqrt{\Delta t_{1}}-1}{S_{0}^{2} \sigma^{2} \Delta t_{1}} \\
\hat{\nu}_{l}=e^{-r t} \max (\bar{S}-K, 0) \sum_{i=1}^{m}\left(-d_{i} \frac{\partial d_{i}}{\partial \sigma}-\frac{1}{\sigma}\right) \\
\hat{\rho}_{l}=e^{-r t} \max (\bar{S}-K, 0)\left(-T+\sum_{i=1}^{m} \frac{d_{i} \sqrt{\Delta t_{i}}}{\sigma}\right)
\end{gathered}
$$

where $\Delta t_{i}=t_{i}-t_{i-1}$, and $d_{i}=\left(\ln \left(S_{i} / S_{i-1}\right)-\left(r-\frac{1}{2} \sigma^{2}\right) \Delta t_{i}\right) /\left(\sigma \sqrt{\Delta t_{i}}\right)$, furthermore, $\partial d_{i} / \partial \sigma=\left(\ln \left(S_{i} / S_{i-1}\right)+\left(r+\frac{1}{2} \sigma^{2}\right) \Delta t_{i}\right) /\left(\sigma^{2} \sqrt{\Delta t_{i}}\right)$,

\section{Monte Carlo method and Antithetic vari- ates}

Thanks to its flexibility, the MC approach had success in finance. Two principal theorems build the main idea of this method, namely, the "Large Numbers Theorem" to estimate the expectation $\mathbb{E}\left(Y_{1}\right)$ by the quantity $\lim _{n \rightarrow \infty} \frac{1}{N} \sum_{n=1}^{N} Y_{n}$ and the "Central Limit Theorem" for obtain confidence interval containing $\mathbb{E}\left(Y_{1}\right)$ for a fixed probability, where $\left(Y_{i}\right)_{i \geq 1}$ is a sequence of independent identically distributed (i.i.d) real square-integrable random variables. The rate convergence of MC methods is typically $N^{-1 / 2}$.

Generally, MC method is composed of three capital steps:

1. simulate sample paths of the value which one wants to estimate,

2. re-simulate $N$ independent reproductions of this value,

3. take the average of all returns. 
let $[0, T]$ be the time interval in which evolves our asset. We subdivide this interval into $m$ points $t_{i}=i T / m$, which are equally-spaced. Form the caracteristics of Brownien Motion, the Black and Scholes formula contained in equation (2) can be written:

$$
S_{i}=S_{i-1} \exp (\mu h+\sigma U \sqrt{h}), \quad i=1, \cdots, m
$$

where $\mu=r-\frac{\sigma^{2}}{2}, S_{i}$ denotes the underlying asset price at date $t_{i}$, and $U$ is a random variable taken from the standard normal distribution.

This formula will be used to compute prices which serve to estimate greeks with finite difference method. Morever, it is necessary to calculate the others estimators. For generating random variables following standard normal distribution, we will use the quadratic Pseudo Random Numbers Generator $($ PRNG)(see Moussi el al. [3]).

In what follow, we expose a method for increasing the efficiency of Monte Carlo simulation by reducing the variance of simulation estimates.

Indeed, The method of antithetic variates attempts to reduce variance by introducing negative dependence between pairs of replications. It is the most widely used techniques in financial problems.

Remark that if $U$ is uniformly distributed over $[0,1]$, then $1-U$ is too. Hence, if we generate a path using as inputs $U_{1}, \cdots, U_{n}$, we can generate a second path using $1-U_{1}, \cdots, 1-U_{n}$ without changing the law of the simulated process. In a simulation driven by independent standard normal random variables, the method of antithetic variates is based on the observation that if $Z_{i}$ has a standard normal distribution, then so does $-Z_{i}$. Then, antithetic variates can be implemented by pairing a sequence $Z_{1}, Z_{2}, \cdots$ of i.i.d. $\mathcal{N}(0,1)$ variables with the sequence $-Z_{1},-Z_{2}, \cdots$ of i.i.d. $\mathcal{N}(0,1)$ variables. If the $Z_{i}$ are used to simulate the increments of a Brownian path, then the $-Z_{i}$ simulate the increments of the reflection of the path about the origin [15].

\section{Numerical results}

Due to its flexibility relative to the choice of parameters, its accuracy, and its compatibility with this kind of simulations. The quadratic PRNG will be used in all simulations [3]. Hence, we can extract a reduced centered normal law variate $\mathcal{N}(0,1)$ from $X_{n}$, by Box-Muller method (see[9]). For speed of execution we implemented all of the simulations in $\mathrm{C}$ programming language. In this paper, we consider The discretely sampled arithmetic Asian option

which uses the average value of the underlying asset at predetermined dates $t_{1}, \ldots, t_{m}$

$$
A_{m}=\frac{1}{m} \sum_{i=1}^{m} S_{t_{i}}
$$


The price is computed by using equation (3). Note that price and sensitivities of continuously sampled Asian options can be estimated by taking sufficiently large values of $m$. However one must account for the inherent discretization bias resulting from the approximation of continuous time processes through discrete sampling. In all simulations $m$ represents number of readings and $M$ replications of sample paths (Monte Carlo loops). The results that we give, the error considered is the standard error which can be defined as $\frac{\sigma}{\sqrt{M}}$, when $\sigma$ is the standard deviation of estimator.

\subsection{Forward finite difference and the central difference}

We first compare forward and central finite difference for delta estimation (with and without common random numbers).

Parameters: $r=0.1, K=100, \sigma=0.25, T=0.2, S_{0}=90$.

we set $m=30$ and vary the value of $M$ (Monte Carlo loop). Forward Findep $_{\text {and }}$

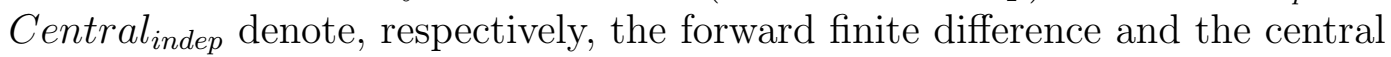
finite difference with independents random variables. Otherwise, Forward Com $_{\text {. }}$ and Central $_{\text {Com }}$ represent, respectively, the forward finite difference and the central finite difference with common random numbers. The four methods are used in accordance with the optimal rate of convergence for each (see [15]). Standard errors related to the use of four approaches are presented in the table 2.

Table 1: Values of Delta in terms of $M$

\begin{tabular}{|l||c|c|c|c|c|}
\hline & $M=100$ & $M=1000$ & $M=10000$ & $M=100000$ & $M=1000000$ \\
\hline Forward $_{\text {indep }}$ & 0.2173 & 0.1892 & 0.1119 & 0.0988 & 0.0948 \\
\hline Forward $_{\text {Com }}$ & 0.1038 & 0.1064 & 0.1007 & 0.0944 & 0.0945 \\
\hline Central $_{\text {indep }}$ & 0.1493 & 0.1331 & 0.0899 & 0.0842 & 0.0823 \\
\hline Central $_{\text {Com }}$ & 0.0925 & 0.0917 & 0.0874 & 0.0820 & 0.0821 \\
\hline
\end{tabular}

Table 2: Standard errors in terms of $M$

\begin{tabular}{|c||c|c|c|c|c|}
\hline & $M=100$ & $M=1000$ & $M=10000$ & $M=100000$ & $M=100000$ \\
\hline Forward $_{\text {indep }}$ & $5.41 \times 10^{-02}$ & $7.91 \times 10^{-03}$ & $1.24 \times 10^{-03}$ & $3.17 \times 10^{-04}$ & $9.50 \times 10^{-05}$ \\
\hline Forward $_{\text {Com }}$ & $1.73 \times 10^{-02}$ & $3.62 \times 10^{-03}$ & $1.02 \times 10^{-03}$ & $2.99 \times 10^{-04}$ & $9.50 \times 10^{-05}$ \\
\hline Central $_{\text {indep }}$ & $4.57 \times 10^{-02}$ & $6.17 \times 10^{-03}$ & $1.02 \times 10^{-03}$ & $2.70 \times 10^{-04}$ & $8.20 \times 10^{-05}$ \\
\hline Central $_{\text {Com }}$ & $3.07 \times 10^{-02}$ & $4.11 \times 10^{-03}$ & $9.20 \times 10^{-04}$ & $2.61 \times 10^{-04}$ & $8.20 \times 10^{-05}$ \\
\hline
\end{tabular}

In order to reflect the behavior of the above methods, we plot the results obtained in the two tables, which gives us the figures below. 

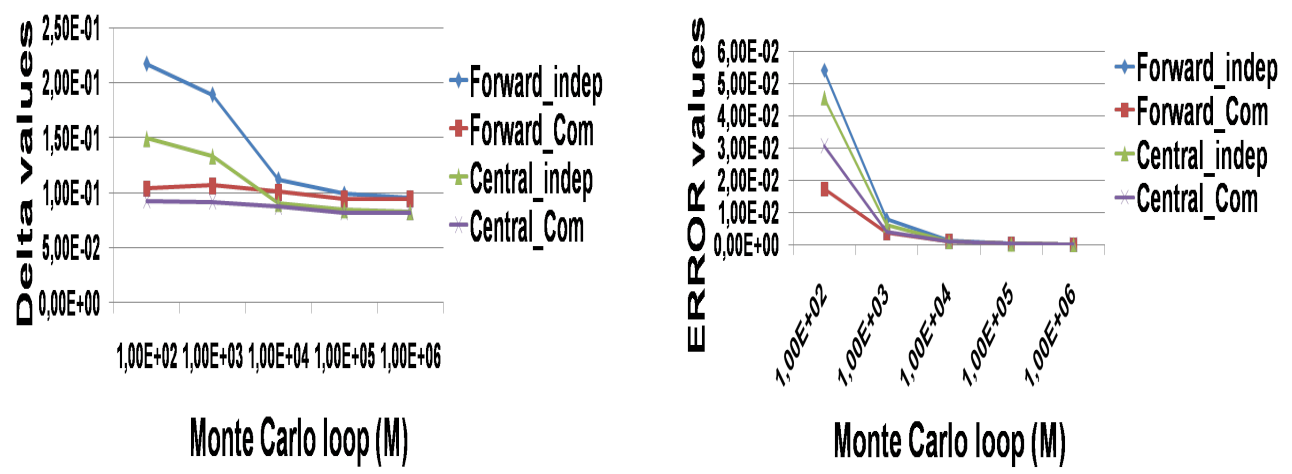

The graph on the left shows that the theoretical convergence rate are audited. Thus it is found that the method of forward finite difference with independents random variables is the slower, the faster is the finite difference by using common ones. Nevertheless, we note that once $M$ is very large (here $M=1000000$ ), the four methods are converging toward "almost" to the same value. This result is confirmed with the right graph, which shows that if $M$ is very large, the methods give the same standard error.

Now, to highlight the effect of the discretization, we take three values of $m$ (number of readings), precisely, $m=30,300,1000$ and set $M=100000$. Results are presented in the following graphs.
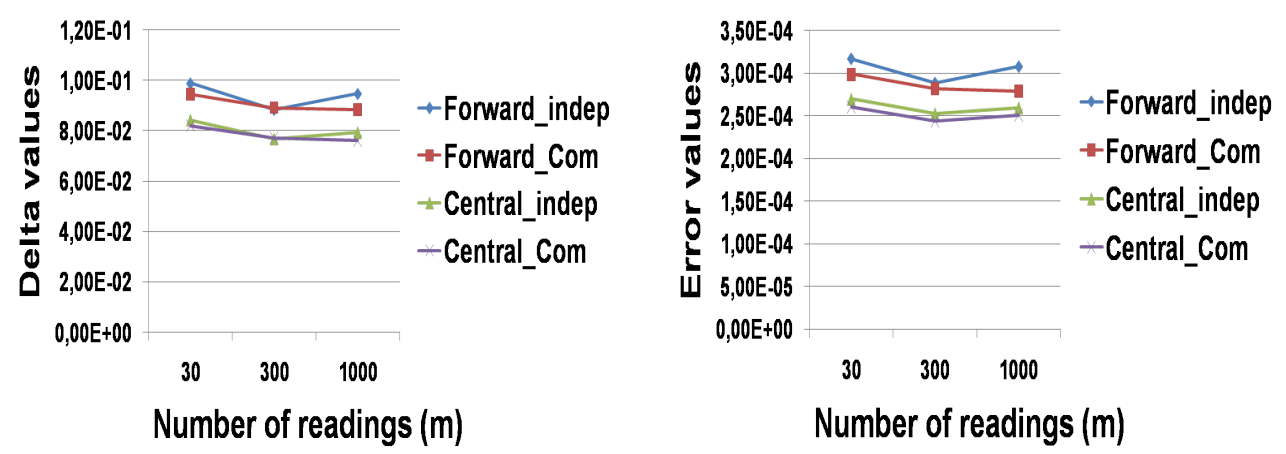

The two graphs show that the method of the approach of central finite difference is less affected by the change in number of readings. Further, generally, more we increase the number of points of discretization, the accuracy is increased except for the method of forward finite difference with independent random variables, which seems unstable depending on the change in number of points of discretization.

\subsection{Greeks of Asian option with and without variance reduction}

In this part, we consider the estimators presented in section 2. In fact, for finite difference method, we explained the general idea. Concerning the Likelihood 
ratio approach, the following estimators are used : $\hat{\Delta}_{l}, \hat{\Gamma}_{l}, \hat{\nu}_{l}$, and $\hat{\rho}_{l}$. Finally, for pathwise method, we use $\hat{\Delta}_{p}$ for estimating delta. For the gamma and rho we employ, respectively, $\hat{\Gamma}_{h}$ the hybrid estimator presented in [13] and $\hat{\nu}_{p}$. For rho note that the results obtained using $\hat{\rho}_{p}$ are unsatisfactory so, we use the estimator $\hat{\rho}_{b}$.

The following table summarizes all results obtained by taking the same option described above with the parameters $r=0.1, K=100, \sigma=0.25, T=$ $0.5, \quad M=50000, m=500$, , changing the value of $S_{0}$. Note that for calculating $\rho$ using finite difference method we take $\epsilon=0.0001$.

Note that for all tables $D F, P W$, and Likelihood denote, respectively, finite difference estimation, pathwise and likelihood ratio methods.

Table 3: Values of greeks and standard errors

\begin{tabular}{|c|c|c|c|c|c|c|}
\hline & \multicolumn{2}{|c|}{$S_{0}=90$} & \multicolumn{2}{|c|}{$S_{0}=100$} & \multicolumn{2}{|c|}{$S_{0}=105$} \\
\hline & Value & Error & Value & Error & Value & Error \\
\hline Delta & & & & & & \\
\hline$F D$ & 0.22570 & $1,010 \times 10^{-03}$ & 0.59912 & $2,679 \times 10^{-03}$ & 0.76011 & $3,398 \times 10^{-03}$ \\
\hline$P W$ & 0.22550 & $1,009 \times 10^{-03}$ & 0.59898 & $2,678 \times 10^{-03}$ & 0.76072 & $3,401 \times 10^{-03}$ \\
\hline Likelihood & 0.21113 & $9,690 \times 10^{-04}$ & 0.58800 & $2,702 \times 10^{-03}$ & 0.76145 & $3,506 \times 10^{-03}$ \\
\hline Gamma & & & & & & \\
\hline$F D$ & 0.31134 & $1,390 \times 10^{-04}$ & 0.03730 & $1,680 \times 10^{-04}$ & 0.02712 & $1,210 \times 10^{-04}$ \\
\hline$P W$ & 0.03230 & $1,460 \times 10^{-04}$ & 0.03684 & $1,660 \times 10^{-04}$ & 0.02566 & $1,150 \times 10^{-04}$ \\
\hline $\begin{array}{c}\text { Likelihood } \\
\text { Rho }\end{array}$ & 0.02282 & $4,530 \times 10^{-04}$ & 0.02340 & $1,057 \times 10^{-03}$ & 0.01887 & $1,303 \times 10^{-03}$ \\
\hline$F D$ & 4.66913 & $4,188 \times 10^{-02}$ & 12.66914 & $4,930 \times 10^{-02}$ & 15.99043 & $4,213 \times 10^{-02}$ \\
\hline$P W$ & 4.28253 & $1,916 \times 10^{-02}$ & 12.01245 & $5,371 \times 10^{-02}$ & 15.30865 & $6,845 \times 10^{-02}$ \\
\hline $\begin{array}{c}\text { Likelihood } \\
\text { Vega }\end{array}$ & 4.64600 & $2,078 \times 10^{-02}$ & 12.57021 & $5,622 \times 10^{-02}$ & 15.81224 & $7,072 \times 10^{-02}$ \\
\hline$\frac{O}{F D}$ & 11.07980 & $4,956 \times 10^{-02}$ & 15.13080 & $6,766 \times 10^{-02}$ & 12.17077 & $5,444 \times 10^{-02}$ \\
\hline$P W$ & 11.07959 & $4,955 \times 10^{-02}$ & 15.13088 & $6,766 \times 10^{-02}$ & 12.16922 & $5,444 \times 10^{-02}$ \\
\hline Likelihood & 8.92731 & $4,514 \times 10^{-02}$ & 12.82907 & $8,673 \times 10^{-02}$ & 10.65001 & $1,039 \times 10^{-02}$ \\
\hline
\end{tabular}

The results of this table show that, although estimators by finite difference are biased and suffer from two shortcomings (discretization and Monte Carlo errors). By choosing optimally the settings, the method succeeds in producing satisfactory results and close to those obtained by the use of the pathwise method for all greeks. Otherwise, the method of likelihood ratio (although estimators are not biased) gives results far from the other two methods, especially for the calculation of gamma and vega (in bold in the table). More generally, this last induces a large error compared with the other two approaches.

The gap that we have between the estimators of gamma and vega by the method of likelihood ratio (in bold in the table) and those by the other two methods can be explained by the fact that the likelihood ratio method is based on the relationship between the probability density of the price of the underlying security and the parameter of interest. Thus if the simulated probability density (drawn by using a PRNG and Box-Muller processing) is far from the theoretical probability density of price, these results are a consequence. 
In the following two tables, we present the results related to the application of two methods for reducing Monte Carlo variance by introducing an antithetic variates. Indeed, in Section (3), we proposed to reduce the variance by transforming the uniform random variable resulting from PRNG (called variance reduction 1), or reduce it by introducing a negative dependence between pairs of replications by using the inverse of Gaussian random variable $Z$ (ie : $-Z$ ) (noted variance reduction 2 ).

Table 4: Values of greeks (Variance Reduction 1) and standard errors

\begin{tabular}{|c|c|c|c|c|c|c|}
\hline & \multicolumn{2}{|c|}{$S_{0}=90$} & \multicolumn{2}{|c|}{$S_{0}=100$} & \multicolumn{2}{|c|}{$S_{0}=105$} \\
\hline & Value & Error & Value & Error & Value & Error \\
\hline Delta & & & & & & \\
\hline$F$ & 0.22360 & $1,000 \times 10^{-03}$ & 0.59571 & $2,664 \times 10^{-03}$ & 0.75817 & $3,390 \times 10^{-03}$ \\
\hline$P W$ & 0.22529 & $1,008 \times 10^{-03}$ & 0.59894 & $2,680 \times 10^{-03}$ & 0.75912 & $3,394 \times 10^{-03}$ \\
\hline Likelihood & 0.21089 & $9,680 \times 10^{-04}$ & 0.58774 & $2,701 \times 10^{-03}$ & 0.76121 & $3,505 \times 10^{-03}$ \\
\hline Gamma & & & & & & \\
\hline$F D$ & 0.03110 & $1,370 \times 10^{-04}$ & 0.03723 & $1,670 \times 10^{-04}$ & 0.02708 & $1,210 \times 10^{-04}$ \\
\hline $\begin{array}{c}P W \\
\text { Rho }\end{array}$ & 0.03230 & $1,460 \times 10^{-04}$ & 0.03639 & $1,630 \times 10^{-04}$ & 0.02486 & $1,110 \times 10^{-04}$ \\
\hline$\frac{F D}{F D}$ & 4.65648 & $4,184 \times 10^{-02}$ & 12.61560 & $4,920 \times 10^{-02}$ & 15.95189 & $4,199 \times 10^{-02}$ \\
\hline$P W$ & 4.23536 & $1,894 \times 10^{-02}$ & 11.95433 & $5,345 \times 10^{-02}$ & 15.29274 & $6,837 \times 10^{-02}$ \\
\hline $\begin{array}{c}\text { Likelihood } \\
\text { Vega }\end{array}$ & 4.63920 & $2,075 \times 10^{-02}$ & 12.56005 & $5,617 \times 10^{-02}$ & 15.79970 & $7,066 \times 10^{-02}$ \\
\hline$F D$ & 11.03678 & $4,936 \times 10^{-02}$ & 15.09398 & $4,936 \times 10^{-02}$ & 12.12296 & $5,423 \times 10^{-02}$ \\
\hline$P W$ & 11.03593 & $4,936 \times 10^{-02}$ & 15.09383 & $6,750 \times 10^{-02}$ & 12.12070 & $5,422 \times 10^{-02}$ \\
\hline
\end{tabular}

Table 5: Values of greeks (Variance Reduction 2) and standard errors

\begin{tabular}{|c|c|c|c|c|c|c|}
\hline & \multicolumn{2}{|c|}{$S_{0}=90$} & \multicolumn{2}{|c|}{$S_{0}=100$} & \multicolumn{2}{|c|}{$S_{0}^{\prime}=105$} \\
\hline & Value & Error & Value & Error & Value & Error \\
\hline \multicolumn{7}{|l|}{ Delta } \\
\hline$F D$ & 0.22347 & $9,990 \times 10^{-04}$ & 0.59576 & $2,664 \times 10^{-03}$ & 0.75829 & $3,390 \times 10^{-03}$ \\
\hline$P W$ & 0.22321 & $9,980 \times 10^{-04}$ & 0.59593 & $2,664 \times 10^{-03}$ & 0.75919 & $3,394 \times 10^{-03}$ \\
\hline Likelihood & 0.21004 & $9,640 \times 10^{-04}$ & 0.58708 & $2,698 \times 10^{-03}$ & 0.76054 & $3,502 \times 10^{-03}$ \\
\hline \multicolumn{7}{|l|}{ Gamma } \\
\hline$F D$ & 0.03099 & $1,310 \times 10^{-04}$ & 0.03725 & $1,670 \times 10^{-04}$ & 0.02707 & $1,210 \times 10^{-04}$ \\
\hline$P W$ & 0.03230 & $1,460 \times 10^{-04}$ & 0.03725 & $1,650 \times 10^{-04}$ & 0.02518 & $1,130 \times 10^{-04}$ \\
\hline \multicolumn{7}{|l|}{ Rho } \\
\hline$F D$ & 4.66913 & $4,188 \times 10^{-02}$ & 12.62839 & $4,929 \times 10^{-02}$ & 15.96573 & $4,207 \times 10^{-02}$ \\
\hline$P W$ & 4.23430 & $1,894 \times 10^{-02}$ & 11.95663 & $5,346 \times 10^{-02}$ & 15.29545 & $6,839 \times 10^{-02}$ \\
\hline Likelihood & 4.61373 & $2,064 \times 10^{-02}$ & 12.52198 & $5,600 \times 10^{-02}$ & 15.75311 & $7,046 \times 10^{-02}$ \\
\hline \multicolumn{7}{|l|}{ Vega } \\
\hline$F D$ & 11.02904 & $4,933 \times 10^{-02}$ & 15.08365 & $6,745 \times 10^{-02}$ & 12.11261 & $5,418 \times 10^{-02}$ \\
\hline$P W$ & 11.02724 & $4,932 \times 10^{-02}$ & 15.08361 & $1,650 \times 10^{-04}$ & 12.11038 & $5,417 \times 10^{-02}$ \\
\hline
\end{tabular}

The results of the two tables show that the calculation of greeks by Monte Carlo method using the three methods chosen can be improved by using the antithetic variates. We see that the outputs are improved and the standard errors are clearly reduced with a slight advantage for the method of variance 
reduction 2 (by introducing $-Z$ ). To account for this effect, we draw the graph below.

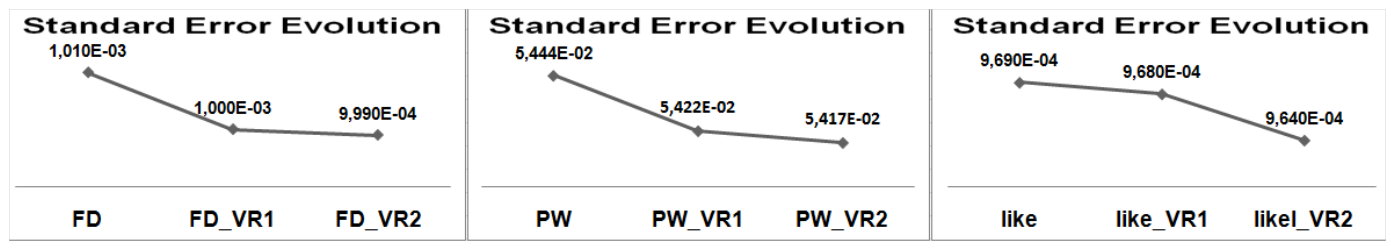

Figure 1: Exemples of the effect of variance reduction techniques

This figure shows the effectiveness of proposed methods to reduce the variance (and therefore the error) of Monte Carlo applied to estimators of sensitivities (greeks) of an arithmetic Asian option.

\section{Conclusion}

In this paper we analyzed three methods of calculating Greek of an Asian option, namely, the finite difference method, the pathwise method, and the likelihood ratio. We first studied different approaches related to the method of finite differences. Indeed, we showed that if the number of replications monte carlo used is very large, so the forward finite difference (with common or independent random variables) and the central one (with common or independent random variables) give almost the same results.

furthermore, we found that the finite difference method with optimally chosen settings produces comparable values to those obtained by the pathwise method. likelihood ratio method generates a larger error than previous approaches and may become unstable.

The results obtained by the three methods have been improved and Monte Carlo error reduced by introducing an antithetic variate. We further showed that, in this context, introducing negative dependence between pairs of replications based on the observation that if $Z_{i}$ has a standard normal distribution, then so does $-Z_{i}$ is the most efficient.

\section{References}

[1] A.G.Z.Kemna, A.C.F.Vorst, A pricing method for options based on average asset values, Journal of Banking Finance, March (1990).

[2] A. Kohatsu-Higa, M. Montero, Malliavin calculus in finance, In handbook of Computational and Numerical Methods in Finance. Birkhauser (2004), 111-174. 
[3] A.Moussi, A.Lidouh and F.Z.Nqi, Simulation of Asian Options Delta Using a Quadratic Congruential Pseudo-Random Numbers Generator, Applied Mathematical Sciences, Vol. 7, no. 93(2013), 4617 - 4629.

[4] B. Lapeyre, E. Temam, Competitive Monte Carlo methods for pricing of Asian Options, Journal of Computational Finance, 5 (1) (2001), 39-57.

[5] E. Benhamou, An application of Malliavin calculus to continuous time Asian options greeks, Technical report. London School of Economics, (2000).

[6] E. Fourni, J.M. Lasry, J. Lebuchoux, P.L. Lions, N. Touzi, Application of Malliavin calculus to Monte Carlo methods in finance, Finance and Stochastics, 3(1999), 391-412.

[7] E. Fourni, J.M. Lasry, J. Lebuchoux, P.L. Lions, Application of Malliavin calculus to Monte Carlo methods in finance.II, Finance and Stochastics, 5(2001), 201-236.

[8] F. Boyle, P.Boyle, Derivatives: The Tools that changed Finance, Risk Books, (2001).

[9] G. E. P. Box, Mervin E. Muller, A Note on the Generation of Random Normal Deviates, The Annals of Mathematical Statistics, Vol. 29, No. 2 (1958), 610-611.

[10] J.E. Zhang, A semi-analytical method for pricing and hedging continuously sampled arithmetic average rate options, Jourual of Computational Finance, 5(2001), 59-79.

[11] J.E. Zhang, Pricing continuously sampled Asian options with perturbation method, Journal of Futures Markets, 23(2003), 535- 560.

[12] J. Hull, Options, Futures, and Other Derivative, Prentice-Hall, 5th ed, 744, July 3 (2002).

[13] M. Broadie, P. Glasserman, Estimating security price derivatives using simulation, Management Science, 42, (1996), 269-285.

[14] N. Ju, Pricing Asian and basket options via Taylor expansion, Journal of Computational Finance, 5 (2002), 79-103.

[15] P. Glasserman, Monte Carlo Methods in Financial Engineering, SpringerVerlag New York, Aout 7 (2003). 
[16] P. Boyle, M. broadie and P. Glasserman, Monte carlo methods for security pricing, Journal of Economic Dynamics and Control, 21(1997), 1267-1321.

[17] P.P. Boyle, A. Potapchik, Prices and sensitivities of Asian options: Asurvey, Insurance: Mathematics and Economics, 42(2008), 189-211.

[18] P.W. Glynn, Optimization of stochastic systems via simulation, In : Proceedings of the 1989 Winter simulation Conference. Society for Computer Simulation, San Diego, (1989), 90-105.

[19] R. Kaas, J. Dhaene and M.J. Goovaerts, Upper and lower bounds for sums of random variables, Insurance: Mathematics and Economics, 27(2000), 151-168.

Received: March 26, 2014 